Adherence to local trust guidance was evident with regards choice of antimicrobial in 59\%. The indication was recorded on the drug chart in $100 \%$, with a review date in $35 \%$ and a stop date in $86 \%$.

Advance discussions relating to antimicrobial treatment were documented in $48 \%$. Seventeen patients died during the admission; the average time between stopping antimicrobials and death was 8 days.

Conclusions This audit highlights the importance of accessing updated guidelines which ensure appropriate prescribing whilst considering local resistance patterns. Alongside a drive to raise awareness, an advance care planning template was implemented for all patients, to record discussions and guide decision making when indications of infection arise. The continuing challenge lies in identifying patients who will benefit from the right antimicrobial and those for whom there will be minimal benefit, but could contribute to increasing antimicrobial resistance.

\section{MEASURING SERUM-ASCITES ALBUMIN GRADIENT TO OPTIMIZE MANAGEMENT OF ASCITES IN A HOSPICE SETTING}

Katherine Bevan, Claire Stark-Toller. Mountbatten Hampshire

\subsection{6/spcare-2021-PCC.148}

Background Paracentesis for management of ascites is frequently performed in hospices. Serum-ascites albumin gradient (SAAG) should be measured to guide management. Patients with ascites caused by portal hypertension have a SAAG $11 \mathrm{~g} /$ L. Usual management is to:

- give human albumin solution (HAS) for renal-protective effects

- prescribe spironolactone to reduce re-accumulation of ascites

This practice is established in the management of portal hypertension due to cirrhosis. However, the incidence of portal hypertension in patients requiring paracentesis for malignant ascites is unknown.

Methods In 2019 guidelines were written to ensure SAAG was checked for patients admitted to the hospice for paracentesis. These guidelines were audited in 2020. The audit covered an 11-month period, looking at whether SAAG was available preprocedure, and whether HAS and spironolactone were given.

Results 25 drains were inserted in 12 different patients. 100\% of these patients had SAAG calculated.

$67 \%(n=8)$ received HAS. 50\% of this group $(n=4)$ had a diagnosis of cirrhosis, and 50\% $(n=4)$ had metastatic malignancy. 3 of the patients given HAS were already on spironolactone, 3 were started on it post procedure (these were patients with malignant ascites), and 1 had their dose increased.

4 patients with malignant ascites received HAS and 3 were started on spironolactone.

Conclusions Measuring SAAG demonstrated cases of malignant ascites associated with portal hypertension. This led to a change in management with increased use of HAS and spironolactone in patients with malignant ascites. All patients undergoing abdominal paracentesis should have SAAG recorded and used to determine appropriate use of HAS and spironolactone.

\section{MYOTONIC DYSTROPHY: THE INTERFACE WITH PALLIATIVE MEDICINE, A SERIES OF CASES}

Laura Dewhirst, Claire Bassie, Ceri Young, Derek Willis. Severn Hospice, Robert Jones Agnes Hunt Orthopaedic Hospital NHS Foundation Trust

\subsection{6/spcare-2021-PCC.149}

Background Myotonic Dsytrophy (DM) is a multi-system disease that affects muscle function. DM is the most common muscular dystrophy occurring in adulthood and the prevalence is around 10 cases per 100,000. There are two distinct forms Type 1 and Type 2. DM predominantly causes weakness of the voluntary muscles but involuntary muscles can also be affected. As the disease progresses respiratory, cardiac and gastrointestinal symptoms can arise. Despite treatment options for management of complications, DM remains a progressive life limiting condition. Severn Hospice is now affiliated with Robert Jones Agnes Hunt Hospital in Oswestry to provide a symptom control clinic for DM patients; something that is not routinely done nationally. Palliative Care should be involved early in the diagnosis to allow more effective symptom control and discussions around Advance Care Planning.

Methods \& Results We collated the data for the 5 patients with a DM diagnosis that were referred to Severn Hospice and/or joint symptom control clinics. All 5 patients were assessed for symptoms that included but were not limited to: breathlessness, pain, dysphagia and low mood. $3 / 5$ patients are still alive and have ongoing support. We coordinated referrals to other specialities including PEG/Respiratory teams for ongoing assessment. 1 patient was admitted to the inpatient unit which allowed for symptoms to be optimised and Advance Care Planning to occur.

Conclusions The role of Palliative Care in the management of DM patients is paramount and early involvement in the disease trajectory is optimal for improving symptom control and providing opportunities for advance care planning relating to specific complications, e.g. cardiac arrhythmias/feeding tube insertion/respiratory support. Clinical guidelines for the role of Palliative Care in DM are limited, despite the fact it is a life limiting illness. This has prompted a focus group who are looking to put together some National Guidance moving forwards.

\section{DO ANTIBIOTICS IMPROVE THE IPOS REPORTED BURDEN OF SYMPTOMS OF PAIN, BREATHLESSNESS AND CONFUSION?}

Lucy Taylor, Sanjay Shah. University Hospitals Leicester, University of Leicester, Northamptonshire Healthcare NHS Trust

\subsection{6/spcare-2021-PCC.150}

Introduction The Integrated Palliative Care Outcome Scale (IPOS) offers potential to measure outcomes of palliative care interventions. ${ }^{1}$ Antibiotics are commonly given for symptomatic relief in hospices but the evidence base is weak, supported typically from the perceived improvement in symptoms as judged by healthcare professionals. ${ }^{2-5}$ IPOS adds the patient's voice to this debate.

Methods A retrospective notes review of 31 inpatients at 2 hospices in Northamptonshire from 20/17/19 to 20/11/19 